\title{
FINITE OPERATORS AND SIMILARITY ORBITS
}

\author{
LAWRENCE A. FIALKOW AND DOMINGO A. HERRERO ${ }^{1}$
}

\begin{abstract}
In this note we answer the question of J. P. Williams as to which Hilbert space operators $T$ have the property that every similarity transformation $W^{-1} T W$ is a finite operator: $T$ has this property if and only if its image in the Calkin algebra satisfies a quadratic equation.
\end{abstract}

1. Introduction. Let $\mathscr{H}$ denote a complex Hilbert space and let $\mathscr{L}(\mathscr{H})$ denote the algebra of all bounded linear operators on $\mathscr{H}$. An operator $T$ in $\mathscr{L}(\mathscr{H})$ is a finite operator if $0 \in W(T X-X T)^{-}$(the closure of the numerical range of $T X-X T$ ) for each $X \in \mathscr{L}(\mathscr{H})$. The concept of a finite operator was introduced by J. P. Williams [25], who studied criteria for finiteness and posed several questions in this context. In the sequel, for the case when $\mathscr{H}$ is separable, we will answer the following question of J. P. Williams [25, Question 3]: For which finite operators $T$ is every similarity transformation $W T W^{-1}$ also finite?

In [25] J. P. Williams proved that $\mathscr{F}(\mathscr{H}) \equiv\{R \in \mathscr{L}(\mathscr{H}): R$ is a finite operator $\}$ is a closed subset of $\mathscr{L}(\mathscr{H})$ that contains each operator having a finite-dimensional reducing subspace (see Theorem 2.3 below). From this fact it follows that $\mathscr{F}(\mathscr{H})$ (or, more simply, $\mathscr{F})$ contains the set $(\mathrm{QD})$ of all quasidiagonal operators in $\mathscr{L}(\mathscr{H})$ (defined below). Let $\mathscr{K}(\mathscr{H})$ denote the ideal of all compact operators in $\mathscr{L}(\mathscr{H})$ and let $\mathscr{A}(\mathscr{H})=\mathscr{L}(\mathscr{H}) / \mathscr{K}(\mathscr{H})$ denote the Calkin algebra; for $T$ in $\mathscr{L}(\mathscr{H})$, we denote by $\tilde{T}$ the image of $T$ in $\mathscr{A}(\mathscr{H})$ under the canonical projection. In [25, Theorem 7] J. P. Williams proved that if $T \in \mathscr{L}(\mathscr{H})$ satisfies a (nontrivial) quadratic equation, then $T$ is finite. More generally, it is known that if $\tilde{T}$ satisfies a polynomial $p$ of degree $\leqslant 2$, then $T$ is quasidiagonal $[5,9]$, hence finite. In this case, each operator $S$ similar to $T$ clearly satisfies $p(\tilde{S})=0$, so we conclude that $\mathscr{S}(T) \subset \mathscr{F}$, where $\mathscr{S}(T) \equiv\left\{X^{-1} T X\right.$ : $X \in \mathscr{L}(\mathscr{H})$ is invertible $\}$ is the similarity orbit of $T$. The converse of this observation is also true and thus provides the following answer to J. P. Williams' question.

THEOREM 1.1. For $T \in \mathscr{L}(\mathscr{H})(\mathscr{H}$ separable $)$ the following are equivalent:

(i) $\mathscr{S}(T) \subset \mathscr{F}$;

(ii) $\mathscr{S}(T) \subset(\mathrm{QD})$;

(iii) $\tilde{T}$ satisfies a polynomial of degree $\leqslant 2$.

In [9, Theorem 2.1] D. A. Herrero proved the equivalence of (ii) and (iii), and we have just seen that (iii) implies (i). To prove Theorem 1.1 it thus remains to show

Received by the editors February 17, 1984.

1980 Mathematics Subject Classification. Primary 47A65; Secondary 47A12, 47A66.

${ }^{1}$ Research of both authors partially supported by grants of the National Science Foundation. 
that (i) implies (iii), and this we will accomplish via a sequence of preliminary results in $\$ 2$. $\$ 3$ contains some observations about compact perturbations of finite operators and an update on the status of the other questions raised by J. P. Williams in [25].

The authors wish to thank Professor William Helton of the University of California at San Diego for making possible their visit to U. C. S. D. (summer, 1983), during which this paper was written.

2. Finite operators and similarity orbits. Let $\mathscr{A}$ denote a complex Banach algebra with identity $e$. A state on $\mathscr{A}$ is a functional $f \in \mathscr{A}^{*}$ such that $f(e)=1=\|f\|$. For $x \in \mathscr{A}$, let $W_{0}(x)=\{f(x): f$ is a state on $\mathscr{A}\}$, the numerical range of $x[22,25]$. $W_{0}(x)$ is a compact convex set containing conh $\sigma(x)$, the convex hull of the spectrum of $x[22$, Theorem 1]. For the case $\mathscr{A}=\mathscr{L}(\mathscr{H})$, if $T \in \mathscr{L}(\mathscr{H})$, then $W_{0}(T)=W(T)^{-}$, where $W(T)=\{(T h, h): h \in \mathscr{H},\|h\|=1\}$ (the spacial numerical range of $T)$. An element $a$ is finite if $0 \in W_{0}(a x-x a)$ for each $x \in \mathscr{A} ; \mathscr{F}(\mathscr{A})$ (or $\mathscr{F}$ ) denotes the set of all finite elements of $\mathscr{A}$. For $x \in \mathscr{A}, \sigma(x)$ and $r(x)$ denote, respectively, the spectrum and spectral radius of $x$. For operators, $\sim$ and $\approx$ will denote similarity and unitary equivalence, respectively.

The basic criteria for finiteness are provided by the following result. This was proved in [25] for the operator case, but the results of [25] show that the proof carries over directly to the Banach algebra setting.

Theorem 2.1 [25, Theorem 4]. For $a \in \mathscr{A}$ the following are equivalent:

(i) $a$ is finite, i.e., $0 \in W_{0}(a x-x a)$ for every $x \in \mathscr{A}$;

(ii) $\|a x-x a-e\| \geqslant 1$ for every $x \in \mathscr{A}$;

(iii) there exists a state $f$ on $\mathscr{A}$ such that $f(a x)=f(x a)$ for every $x \in \mathscr{A}$.

In the operator case, for $A \in \mathscr{L}(\mathscr{H})$ we may reformulate the condition $\mathscr{S}(A) \subset \mathscr{F}$ as follows.

Proposition 2.2. For $A \in \mathscr{L}(\mathscr{H}), \mathscr{S}(A) \subset \mathscr{F}$ if and only if $0 \in \operatorname{conh} \sigma(A X-X A)$ for each $X \in \mathscr{L}(\mathscr{H})$.

Proof. Let $\mathscr{G}(\mathscr{H})$ denote the set of all invertible operators in $\mathscr{L}(\mathscr{H})$. For $T \in \mathscr{L}(\mathscr{H})$,

$$
\operatorname{conh} \sigma(T)=\bigcap\left\{W\left(S^{-1} T S\right)^{-}: S \in \mathscr{G}(\mathscr{H})\right\}
$$

[14; 24, p. 308]. Thus $0 \in \operatorname{conh} \sigma(A X-X A)$ for each $X$ in $\mathscr{L}(\mathscr{H})$ if and only if $0 \in W\left(S^{-1} A S S^{-1} X S-S^{-1} X S S^{-1} A S\right)^{-}$for each $X$ in $\mathscr{L}(\mathscr{H})$ and each $S$ in $\mathscr{G}(\mathscr{H})$, i.e., $S^{-1} A S \in \mathscr{F}$ for every $S \in \mathscr{G}(\mathscr{H})$.

We next note for ease of reference some of the criteria for finiteness of operators due to J. P. Williams [25]. For $n \geqslant 1$, let $\mathscr{R}_{n}=\{T \in \mathscr{L}(\mathscr{H}): T$ has an $n$-dimensional reducing subspace $\}$.

TheOREM 2.3. (i) [25, Corollary, p. 133] $\mathscr{F}$ is closed in $\mathscr{L}(\mathscr{H})$;

(ii) [25, Theorem 6] $\mathscr{R}_{n} \subset \mathscr{F}(n \geqslant 1)$;

(iii) if $A \in \mathscr{F}, B \in \mathscr{L}(\mathscr{H})$, then $A \oplus B \in \mathscr{F}$. 
(Statement (iii) follows from the equivalence between (i) and (ii) in Theorem 2.1.)

Recall from [8] that an operator $T \in \mathscr{L}(\mathscr{H})$ is quasidiagonal if there exists an increasing sequence of finite rank projections, $\left\{P_{n}\right\}_{n=1}^{\infty}, P_{n} \rightarrow 1$ (convergence in the strong operator topology), such that $\left\|A P_{n}-P_{n} A\right\| \rightarrow 0 . T$ is block diagonal if, with $\left\{P_{n}\right\}$ as above, $A P_{n}=P_{n} A$ for each $n$. Let (BD) and (QD) denote the sets of all block diagonal and, respectively, quasidiagonal operators in $\mathscr{L}(\mathscr{H})$; thus $(\mathrm{QD})=$ $(\mathrm{BD})+\mathscr{K}(\mathscr{H})$ and $(\mathrm{BD})^{-}=(\mathrm{QD})[8]$. Theorem 2.3 implies that $(\mathrm{QD}) \subset \mathscr{F}$, and the inclusion is proper (see below). An operator $T$ is quasitriangular if there exists an increasing sequence of finite rank projections $\left\{P_{n}\right\}, P_{n} \rightarrow 1$, such that $\left\|\left(1-P_{n}\right) T P_{n}\right\|$ $\rightarrow 0$ [6]. $T$ is biquasitriangular if $T$ and $T^{*}$ are quasitriangular. Let (QT) and (BQT) denote the sets of all quasitriangular and, respectively, biquasitriangular operators in $\mathscr{L}(\mathscr{H})$; thus $(\mathrm{QD}) \subset(\mathrm{BQT}) \subset(\mathrm{QT})$ and all inclusions are proper $[\mathbf{6}, \mathbf{8}, \mathbf{2 0}]$.

Relationships among the various classes of operators just introduced may be further clarified with the help of the following result.

Theorem 2.4 [25, TheOREM 8]. Let $A \in \mathscr{L}(\mathscr{H})$. The operator $T=T(A)$ on $\mathscr{H}^{(3)}$ defined by the matrix

$$
\left(\begin{array}{ccc}
0 & 1 & A \\
0 & 0 & 1 \\
0 & 0 & 0
\end{array}\right)
$$

is finite if and only if $A$ is finite.

In [20] R. Smucker proved the exact analogue of Theorem 2.4 with "finite" replaced by "quasidiagonal", and $\mathrm{R}$. Smucker's result plays a role in the proof of the equivalence of (ii) and (iii) of Theorem 1.1 (see [9, p. 202]). For the moment, let $A$ denote the unilateral shift; then $A$ is finite [25, p. 133] but not quasitriangular [6]. Thus $T(A)$ is finite and nonquasidiagonal; however, $T(A)$ is nilpotent and is thus biquasitriangular [8], i.e., $T(A) \in \mathscr{F} \cap(\mathrm{BQT}) \backslash(\mathrm{QD})$.

Before presenting the next result we require some preliminaries concerning spectral sets. Recall from [16, 24] that a closed subset $\sigma$ of the complex plane is a spectral set of an operator $T$ if $\sigma(T) \subset \sigma$ and $\|r(T)\| \leqslant \sup \{|r(z)|: z \in \sigma\}$ for each rational function $r$ with poles off $\sigma$; in this case $r(\sigma)^{-}$is a spectral set for $r(T)$ (see [24]). We will employ the following facts about spectral sets:

(i) (von Neumann's Inequality [16]) The unit disk is a spectral set for every contraction.

(ii) For $T \in \mathscr{L}(\mathscr{H}), W(T)^{-}$is contained in every convex spectral set of $T$ (see [24, p. 308]).

(iii) Let $R_{+}^{2}=\{\lambda \in \mathrm{C}: \operatorname{Re} \lambda>0\}$. If $T \in \mathscr{L}(\mathscr{H})$ and $W(T)^{-} \subset R_{+}^{2}$, then there is a closed disk $\Delta \subset R_{+}^{2}$ that is a spectral set for $T$. (To see this, recall from [15, Lemma 12] (cf. [22, Lemma 2, p. 419]) that

$$
\lim _{\gamma \rightarrow+\infty}(\|\gamma-T\|-\gamma)=\sup \{\operatorname{Re} \lambda: \lambda \in W(-T)\}=-\inf \{\operatorname{Re} \lambda: \lambda \in W(T)\} .
$$

Since $W(T)^{-} \subset R_{+}^{2}$, it follows that there exist $\gamma, \delta>0$ such that $\|\gamma-T\|-\gamma<-\delta$, whence $\|\gamma-T\|<\gamma-\delta$. Thus the unit disk is a spectral set for the contraction 
$(1 /(\gamma-\delta))(\gamma-T)($ see (i)), and it follows that the disk $\Delta=\{\lambda \in \mathbf{C}:|\lambda-\gamma| \leqslant \gamma$ $-\delta\} \subset R_{+}^{2}$ is a spectral set for $T$.)

For $k \geqslant 1$, let $q_{k}$ denote the Jordan nilpotent $k$-cell acting on $\mathbf{C}^{k}$. For a Hilbert space $\mathscr{K}, T \in \mathscr{L}(\mathscr{K})$ and $\alpha \geqslant 1$, let $\mathscr{K}^{(\alpha)}$ denote the orthogonal direct sum of $\alpha$ copies of $\mathscr{K}$ and let $T^{(\alpha)} \in \mathscr{L}\left(\mathscr{K}^{(\alpha)}\right)$ denote the orthogonal direct sum of $\alpha$ copies of $T$.

LEMMA 2.5. $q_{1} \oplus q_{3}^{(\infty)}$ is similar to an operator that is not finite.

Proof. Observe that for any $A \in \mathscr{L}(\mathscr{H}), M(A)=0_{\mathrm{C}} \oplus T(A)$ is similar to $q_{1} \oplus q_{3}^{(\infty)}$. It thus suffices to exhibit an operator $A$ such that $M=M(A)$ is similar to an operator that is not finite.

Let $A$ denote an operator that is not finite. Theorem 2.4 implies that $T(A) \notin \mathscr{F}$ so, from Theorem 2.1(i), there exists $X \in \mathscr{L}(\mathscr{H})$ such that $0 \notin W(T X-X T)^{-}$. Replacing $X$ by $e^{i t} X$ for a suitable $t>0$, we can directly assume that $W(T X-X T)^{-}$ $\subset R_{+}^{2}$. From (iii) above, there exists a closed disk $\Delta \subset R_{+}^{2}$ that is a spectral set of $T X-X T$. Thus if $\mu \in \mathbf{C}, \operatorname{Re} \mu \leqslant 0$, then the closed disk $\Delta_{\mu}=\left\{(\mu-\lambda)^{-1}: \lambda \in \Delta\right\}$ $\left(\subset \mathbf{C} \backslash\left(R_{+}^{2}\right)^{-}\right)$is a spectral set of $R=R(T, X, \mu) \equiv(\mu-(T X-X T))^{-1}$; in particular, $W(R) \subset \Delta_{\mu}$.

Proposition 2.2 implies that to show $\mathscr{S}(M) \not \subset \mathscr{F}$ it suffices to produce an operator $Y$ such that $\sigma(M Y-Y M) \subset R_{+}^{2}$. Our construction of $Y$ proceeds as follows. Let $e$ be a unit vector in $\mathbf{C}$ and let $f, g \in \mathscr{H}^{(3)}$. Let $F=e \otimes f: \mathscr{H}^{(3)} \rightarrow \mathbf{C}$ and let $G=g \otimes e: \mathbf{C} \rightarrow \mathscr{H}^{(3)}$. Let $Y$ be the operator on $\mathbf{C} \oplus \mathscr{H}^{(3)}$ with operator matrix $\left(\begin{array}{ll}0 & F \\ G & X\end{array}\right)$. We will show that a suitable choice of $f$ and $g$ gives $Y$ the desired property.

The matrix of $M Y-Y M$ is $\left(\begin{array}{c}0 \\ T G T X-X T\end{array}\right)$. Since $\sigma(T X-X T) \subset R_{+}^{2}$, if

$$
\sigma(M Y-Y M) \not \subset R_{+}^{2},
$$

then $M Y-Y M$ has an eigenvalue $\mu$ with $\operatorname{Re} \mu \leqslant 0$. A corresponding (nonzero) eigenvector cannot be of the form $v=0 \oplus w \in \mathbf{C} \oplus \mathscr{H}^{(3)}$, for a matrix calculation of $(M Y-Y M-\mu) v=0$ shows that $((T X-X T)-\mu) w=0$, a contradiction. Thus $M Y-Y M$ has an eigenvector for $\mu$ of the form $e \oplus w$, and a calculation shows that $\mu e=-F T w$, $\because=R T G e$, and thus $\mu e=-F T R T G e$. Now $\mu=(\mu e, e)=$ $-(F T R T G e, e)=-\left(\left(e \otimes T^{*} f\right) R(T g \otimes e) e, e\right)=-\left(R T g, T^{*} f\right)$. Let

$$
f=\left(\begin{array}{l}
f_{1} \\
f_{2} \\
f_{3}
\end{array}\right), \quad g=\left(\begin{array}{l}
g_{1} \\
g_{2} \\
g_{3}
\end{array}\right)
$$

then

$$
T g=\left(\begin{array}{c}
g_{2}+A g_{3} \\
g_{3} \\
0
\end{array}\right) \quad \text { and } \quad T^{*} f=\left(\begin{array}{c}
0 \\
f_{1} \\
A^{*} f_{1}+f_{2}
\end{array}\right)
$$


Let $g_{1}=0$, let $g_{3}$ be a unit vector in $\mathscr{H}$, and let $g_{2}=-A g_{3}$; let $f_{1}=g_{3}, f_{2}=-A^{*} f_{1}$ and $f_{3}=0$. Then $h \equiv T g=T^{*} f$ is a unit vector and thus $\mu=-(R h, h) \in W(-R) \subset$ $R_{+}^{2}$, a contradiction since $\operatorname{Re} \mu \leqslant 0$. We conclude that with $f$ and $g$ as just described, $\sigma(M Y-Y M) \subset R_{+}^{2}$, so the proof is complete.

COROLlaRY 2.6. $q_{1}^{(\infty)} \oplus q_{3}^{(\infty)}$ is similar to an operator that is not finite.

Proof. Let $T=q_{1} \oplus q_{3}^{(\infty)}$ and (using Lemma 2.5) let $S$ be a similarity of $T$ that is not finite. Now $q_{1}^{(\infty)} \oplus q_{3}^{(\infty)} \approx T^{(\infty)} \sim S^{(\infty)}$, and it follows from Theorem 2.1(ii) that $S^{(\infty)}$ is not finite.

REMARK. A cumbersome computation based on the same argument as in the proof of $[25$, Theorem 8$]$ actually shows that

$$
L(X)=\left(\begin{array}{llll}
0 & 0 & 0 & 1 \\
0 & 0 & 1 & X \\
0 & 0 & 0 & 1 \\
0 & 0 & 0 & 0
\end{array}\right) \in \mathscr{L}\left(\mathscr{H}^{(4)}\right)
$$

is finite if and only if $X \in \mathscr{L}(\mathscr{H})$ is finite. Clearly, $L(X)$ is similar to $q_{1}^{(\infty)} \oplus q_{3}^{(\infty)}$.

For $T \in \mathscr{L}(\mathscr{H}), \sigma_{e}(T)=\sigma(\tilde{T})$ denotes the essential spectrum of $T$.

LemMA 2.7. Let $T \in \mathscr{L}(\mathscr{H})$ and suppose $\tilde{T}$ does not satisfy a polynomial of degree $\leqslant 2$. Then there exist (not necessarily distinct!) points $\alpha_{1}, \alpha_{2}, \alpha_{3} \in \sigma_{e}(T)$ such that, for $n \geqslant 1, \mathscr{S}(T)^{-}$contains an operator unitarily equivalent to

$$
A_{n}=\left(\begin{array}{cccc}
T^{\prime} & 0 & 0 & 0 \\
0 & \alpha_{1} & n & 0 \\
0 & 0 & \alpha_{2} & n \\
0 & 0 & 0 & \alpha_{3}
\end{array}\right) \in \mathscr{L}\left(\mathscr{H}^{(4)}\right)
$$

where $T^{\prime}$ is unitarily equivalent to $T \oplus \alpha_{1} 1_{\mathscr{H}}$.

Proof. Choose $\alpha_{1}, \alpha_{2}, \alpha_{3}$ as follows:

(1) If $\sigma_{e}(T)$ contains three distinct points, choose these as $\alpha_{1}, \alpha_{2}, \alpha_{3}$.

(2) If $\sigma_{e}(T)=\{\alpha\}$ is a singleton, then our hypothesis implies that $(\tilde{T}-\alpha)^{2} \neq 0$. Let $\alpha_{1}=\alpha_{2}=\alpha_{3}=\alpha$.

(3) If $\sigma_{e}(T)=\{\alpha, \beta\}$ (two distinct points), then $T$ is similar to $T_{\alpha} \oplus T_{\beta}$, where $\sigma_{e}\left(T_{\alpha}\right)=\{\alpha\}, \sigma_{e}\left(T_{\beta}\right)=\{\beta\}$, and the hypothesis implies that $\tilde{T}_{\alpha} \neq \alpha$ or $\tilde{T}_{\beta} \neq \beta$. In the first case, let $\alpha_{1}=\alpha_{2}=\alpha$ and $\alpha_{3}=\beta$; in the second case, let $\alpha_{1}=\alpha_{2}=\beta$ and $\alpha_{3}=\alpha$.

It follows from [4; 3, Theorem 9.2] that $\mathscr{S}(T)^{-}$contains an operator unitarily equivalent to $A_{n}$.

Proof of Theorem 1.1. It remains to prove (i) $\Rightarrow$ (iii). Let $T \in \mathscr{L}(\mathscr{H})$ and suppose that $\tilde{T}$ does not satisfy a polynomial of degree $\leqslant 2$. Let $\alpha_{1}, \alpha_{2}, \alpha_{3} \in \sigma_{e}(T)$ be points determined by Lemma 2.7, and for each $n \geqslant 1$, let $A_{n} \in \mathscr{L}\left(\mathscr{H}^{(4)}\right)$ denote the operator defined in Lemma 2.7. Thus, for $n \geqslant 1$, there exists an invertible operator $X_{n}: \mathscr{H} \rightarrow \mathscr{H}^{(4)}$ normal such that $\left\|X_{n} T X_{n}^{-1}-A_{n}\right\|<1$. Let $R=q_{1}^{(\infty)} \oplus q_{3}^{(\infty)}$ $\in \mathscr{L}\left(\mathscr{H}^{(\infty)}\right)$ and let $T_{n}=A_{n}-n R$; thus $\left\|T_{n}\right\|=\|T\|$. Lemma 2.6 implies that there 
exists an invertible operator $W \in \mathscr{L}\left(\mathscr{H}^{(4)}\right)$ such that $W R W^{-1}$ is not finite. For $F \in \mathscr{F}\left(\mathscr{H}^{(4)}\right)$ we have

$$
\begin{aligned}
\left\|W X_{n} T X_{n}^{-1} W^{-1}-F\right\| & \geqslant\left\|n W R W^{-1}-F\right\|-\left\|W\left(X_{n}^{-1} T X_{n}-A_{n}\right) W^{-1}\right\|-\left\|W T_{n} W^{-1}\right\| \\
& \geqslant n \cdot \operatorname{dist}\left[W R W^{-1}, \mathscr{F}(\mathscr{H}(4))\right]-\|W\|\left\|W^{-1}\right\|(1+\|T\|) .
\end{aligned}
$$

Since $\operatorname{dist}\left[W R W^{-1}, \mathscr{F}\left(\mathscr{H}^{(4)}\right)\right]>0$, it now follows that for all sufficiently large $n$, $\operatorname{dist}\left[W X_{n} T X_{n}^{-1} W^{-1}, \mathscr{F}\left(\mathscr{H}^{(4)}\right)\right]>0$. If $U: \mathscr{H} \rightarrow \mathscr{H}^{(4)}$ is a unitary operator, then $U^{*} W X_{n} T X_{n}^{-1} W^{-1} U \in \mathscr{S}(T) \backslash \mathscr{F}$; the proof is complete.

We next present an analogue of Theorem 1.1 for elements of the Calkin algebra. For $T \in \mathscr{L}(\mathscr{H}), \mathscr{S}(\tilde{T})$ denotes the similarity orbit of $\tilde{T}$ with respect to the invertible elements of $\mathscr{A}(\mathscr{H})$.

Corollary 2.8. Let $T \in \mathscr{L}(\mathscr{H}) . \mathscr{S}(\tilde{T}) \subset \mathscr{F}(\mathscr{A}(\mathscr{H}))$ if and only if $\tilde{T}$ satisfies a polynomial of degree $\leqslant 2$.

Proof. Since the projection of $\mathscr{L}(\mathscr{H})$ onto the Calkin algebra is norm-decreasing, it follows from Theorem 2.1(ii) that if $\mathscr{S}(\tilde{T}) \subset \mathscr{F}(\mathscr{A}(\mathscr{H}))$, then $\mathscr{S}(T) \subset \mathscr{F}(\mathscr{L}(\mathscr{H})$ ); in this case, Theorem 1.1 implies that $\tilde{T}$ satisfies a polynomial of degree $\leqslant 2$.

Conversely, suppose $p(\tilde{T})=0$ for some polynomial $p$ of degree $\leqslant 2$. Let $\psi: \mathscr{A}(\mathscr{H}) \rightarrow \mathscr{L}\left(\mathscr{H}_{\psi}\right)$ denote an isometric unital $*$-representation. If $\tilde{J} \in \mathscr{A}(\mathscr{H})$ is invertible, then $p\left(\psi\left(\tilde{J}^{-1} \tilde{T} \tilde{J}\right)\right)=\psi\left(\tilde{J}^{-1} p(\tilde{T}) \tilde{J}\right)=0$, so [25, Theorem 7] implies that $\psi\left(\tilde{J}^{-1} \tilde{T} \tilde{J}\right)$ is finite. From Theorem 2.1(ii) we see that for each $\tilde{X} \in \mathscr{A}(\mathscr{H})$,

$$
\left\|\left(\tilde{J}^{-1} \tilde{T} \tilde{J}\right) \tilde{X}-\tilde{X}\left(\tilde{J}^{-1} \tilde{T} \tilde{J}\right)-1\right\|=\left\|\psi\left(\tilde{J}^{-1} \tilde{T} \tilde{J}\right) \psi(\tilde{X})-\psi(\tilde{X}) \psi\left(\tilde{J}^{-1} \tilde{T} \tilde{J}\right)-1\right\| \geqslant 1,
$$

so another application of Theorem 2.1(ii) implies that $\tilde{J}^{-1} \tilde{T} \tilde{J}$ is finite. Thus $\mathscr{S}(\tilde{T}) \subset \mathscr{F}(\mathscr{A}(\mathscr{H}))$ and the proof is complete.

3. The structure of finite operators: complementary results and open problems. In [25] J. P. Williams raised four questions related to the structure of finite operators, the third of which was resolved by Theorem 1.1. We next recount the status of the other questions raised in [25]. For $A \in \mathscr{L}(\mathscr{H})$, let $\delta_{A}: \mathscr{L}(\mathscr{H}) \rightarrow \mathscr{L}(\mathscr{H})$ denote the inner derivation on $\mathscr{L}(\mathscr{H})$ induced by $A$, defined by $\delta_{A}(X)=A X-X A$. A classical result of $[23,26]$ shows that the identity operator is not a commutator, i.e., $1 \notin \operatorname{Ran} \delta_{A}$. On the other hand, J. Anderson [2] proved that there exist operators $A \in \mathscr{L}(\mathscr{H})$ such that $1 \in\left(\operatorname{Ran} \delta_{A}\right)^{-}$(norm closure). Such operators were recently studied by the second-named author in [11]; following [11], let

$$
J A(\mathscr{H})=\left\{A \in \mathscr{L}(\mathscr{H}): 1 \in\left(\operatorname{Ran} \delta_{A}\right)^{-}\right\} .
$$

$J A(\mathscr{H})$ is closed under similarity and clearly $J A(\mathscr{H}) \cap \mathscr{F}=\varnothing$; thus there exist operators $A$ such that no similarity of $A$ is finite, and this answers in the negative [25, Question 2].

Question 3.1. If $T \in J A(\mathscr{H})$ or $T \in \mathscr{L}(\mathscr{H})$ and $\tilde{T}$ satisfies a polynomial of degree $\leqslant 2$, then $\operatorname{dist}\left(\operatorname{Ran} \delta_{W^{-1} T W}, 1\right)(W$ in $\mathscr{G}(\mathscr{H}))$ is independent of $W$. Are there other operators with this property? 
Question 4 of [25] asks whether $\operatorname{Ran} \delta_{A}$ can be norm-dense in $\mathscr{L}(\mathscr{H})$; in [21, Theorem 1] J. G. Stampfli showed that the dimension of $\mathscr{L}(\mathscr{H}) /\left(\operatorname{Ran} \delta_{A}\right)^{-}$is uncountable. Question 1 of [25] asks whether $\bigcup_{n=1}^{\infty} \mathscr{R}_{n}$ is dense in $\mathscr{F}$; this question, perhaps the most important concerning finite operators, remains unsolved.

In the remainder of this section we consider finite elements of the Calkin algebra and compact perturbations of finite operators. In [25] J. P. Williams observed that $\mathscr{F}$ is not closed under compact perturbations; we illustrate this in the following

Example 3.2. For each $n \geqslant 1$, there exist $T \in \mathscr{R}_{n}$ and $F \in \mathscr{L}(\mathscr{H})$, with $\operatorname{rank}(F)$ $\leqslant n$, such that $T+F$ is not finite. Let $A \in J A(\mathscr{H})$ be an invertible operator with $\|A\|<1$ (note that $J A(\mathscr{H})$ is invariant under translation by scalars and multiplication by nonzero scalars). Let $Y \in \mathscr{L}(\mathscr{H})$ satisfy $\|A Y-Y A-1\|<1-\frac{1}{2}(1+\|A\|)$. Let $V: \mathscr{H} \rightarrow \mathbf{C}^{n}$ denote a coisometry and let $T=A \oplus 0_{\mathbf{C}^{n}} \in \mathscr{L}\left(\mathscr{H} \oplus \mathbf{C}^{n}\right)$. Let $S$ and $X$ denote the operators on $\mathscr{H} \oplus \mathbf{C}^{n}$ with operator matrices of the form

$$
S=\left(\begin{array}{ll}
A & 0 \\
V & 0
\end{array}\right) \text { and } X=\left(\begin{array}{cc}
Y & \frac{1}{2} V^{*} \\
V Y A^{-1} & 0
\end{array}\right)
$$

Now $T \in \mathscr{R}_{n}$ is finite, $F=T-S$ has rank $n$, but $S=T-F$ is not finite. Indeed, the matrix of $S X-X S-1$ is of the form

$$
\left(\begin{array}{cc}
A Y-Y A-1-\frac{1}{2} V^{*} V & \frac{1}{2} A V^{*} \\
0 & -\frac{1}{2}
\end{array}\right),
$$

and thus

$$
\begin{aligned}
\|S X-X S-1\| & \leqslant\left(\|A Y-Y A-1\|+\frac{1}{2}\right)+\left\|\frac{1}{2} A V^{*}\right\| \\
& \leqslant\|A Y-Y A-1\|+\frac{1}{2}(1+\|A\|)<1 .
\end{aligned}
$$

In contrast to the preceding example, a finite operator $T$ may satisfy $T+$ $\mathscr{K}(\mathscr{H}) \subset \mathscr{F}$, and we next examine this case. Suppose that $T$ is finite, and let $f$ be the state of Theorem 2.1(iii). Since $f \in \mathscr{L}(\mathscr{H})^{*}$, it admits a unique decomposition of the form $f=f_{t}+f_{s}$, where $f_{t}(X)=\operatorname{trace}(X C)$ for a suitable trace class operator $C, f_{s}$ is a singular functional (that is, $\operatorname{ker} f_{s} \supset \mathscr{K}(\mathscr{H})$ ), and $\|f\|=\left\|f_{t}\right\|+\left\|f_{s}\right\|=1$ (see, e.g. [19, Chapter IV, p. 50]). In [1, Theorem 10.10] J. H. Anderson proved that if $f_{t} \neq 0$, then $T$ has a finite-dimensional reducing subspace. Conversely, if $T \in \mathscr{R}_{n}$ for some $n \geqslant 1$, then it is easy to construct a state $f$ satisfying condition (iii) of Theorem 2.1 such that $f=f_{t}$ (i.e., $f_{s}=0$ ). Combining these observations with the fact that every operator in $\mathscr{L}(\mathscr{H})$ is a compact perturbation of an irreducible operator [7; 10, Lemma 4.33, p. $104]$, we have the following result.

Proposition 3.3. The following are equivalent for $T$ in $\mathscr{L}(\mathscr{H})$ :

(i) $T+\mathscr{K}(\mathscr{H}) \subset \mathscr{F}(\mathscr{H})$.

(ii) There exists $K \in \mathscr{K}(\mathscr{H})$ such that $T+K$ is a finite irreducible operator.

(iii) There exists $C \in \mathscr{K}(\mathscr{H})$ such that $T+C \in \mathscr{F}(\mathscr{H}) \backslash\left(\bigcup_{n=1}^{\infty} \mathscr{R}_{n}\right)$.

(iv) $\tilde{T} \in \mathscr{F}(\mathscr{A}(\mathscr{H}))$.

Proof. We have already observed (in the proof of Corollary 2.8) that (iv) $\Rightarrow$ (i). On the other hand, the implications (i) $\Rightarrow$ (ii) $\Rightarrow$ (iii) follow immediately from the 
preceding remarks. Thus, in order to complete the proof, we only have to show that (iii) $\Rightarrow$ (iv). But this also follows from the previous observations, because if $T+C \in$ $\mathscr{F}(\mathscr{H}) \backslash\left(\cup_{n=1}^{\infty} \mathscr{R}_{n}\right)$ the state $f$ of Theorem 2.1 (iii) (for $T+C$ ) is necessarily singular. Thus $f: \mathscr{L}(\mathscr{H}) \rightarrow \mathrm{C}$ induces a state $\varphi: \mathscr{A}(\mathscr{H}) \rightarrow \mathrm{C}$ defined by $\varphi(\tilde{X})=f(X)$ (i.e., $f$ can be factored through the Calkin algebra), and $\varphi$ satisfies $\varphi(\tilde{X} \tilde{T})=f(X T)=$ $f(T X)=\varphi(\tilde{T} \tilde{X})$ for all $\tilde{X}$ in $\mathscr{A}(\mathscr{H})$, whence we deduce that $\tilde{T} \in \mathscr{F}(\mathscr{A}(\mathscr{H}))$.

It is not difficult to check that every operator $T$ of the form

$$
T=(A \oplus B)+K \in \mathscr{L}(\mathscr{H} \oplus \mathscr{H})
$$

(where $A$ is an arbitrary operator, $B \in \mathscr{L}(\mathscr{H})$ is block diagonal, and $K$ is compact) satisfies the (equivalent) conditions of Proposition 3.3. It was conjectured in [13] that these are the only operators in $\mathscr{L}(\mathscr{H})$ that satisfy the conditions of Proposition 3.3.

The class of operators satisfying $(*)$ is rather large. In particular, it contains (QD), and also every operator $M$ in $\mathscr{L}(\mathscr{H})$ such that $R_{e}^{(n)}(M) \neq \varnothing$ for some $n \geqslant 1$ (see $[17,27]$ ). (Here $R_{e}^{(n)}(M)$ denotes the reducing $n \times n$ essential spectrum of $M$ in the sense of C. Pearcy and N. Salinas [17].) In the Banach algebra setting, it is known that if the norm and spectral radius of an element coincide, then the element is finite (see [25, p. 133]). It is not difficult to show that if an element $\tilde{T}$ of the Calkin algebra has this property and if $\lambda \in \sigma_{e}(T)$ satisfies $|\lambda|=\|\tilde{T}\|$, then $\lambda$ is a normal essential eigenvalue of $T$ in the sense of N. Salinas [18], and thus $\lambda \in R_{e}^{(1)}(T)$ and $T$ satisfies (*).

\section{REFERENCES}

1. J. H. Anderson, Derivations, commutators, and the essential numerical range, Dissertation, Indiana University, 1971.

2. Derivation ranges and the identity, Bull. Amer. Math. Soc. 79 (1973), 705-708.

3. C. Apostol, L. A. Fialkow, D. A. Herrero and D. Voiculescu, Approximation of Hilbert space operators. II, Res. Notes in Math., Pitman, Boston, Mass., 1984.

4. C. Apostol, D. A. Herrero and D. Voiculescu, The closure of the similarity orbit of a Hilbert space operator, Bull. Amer. Math. Soc. (N.S.) 6 (1982), 421-426.

5. L. A. Fialkow, The similarity orbit of a normal operator, Trans. Amer. Math. Soc. 210 (1975), $129-137$.

6. P. R. Halmos, Quasitriangular operators, Acta Sci. Math. (Szeged) 29 (1968), 283-293.

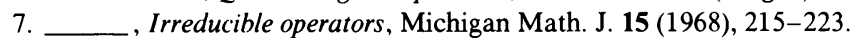

8. __ Ten problems in Hilbert space, Bull. Amer. Math. Soc. 76 (1970), 887-933.

9. D. A. Herrero, Quasidiagonality, similarity, and approximation by nilpotent operators, Indiana Univ. Math. J. 30 (1981), 199-233.

10. __ Approximation of Hilbert space operators. I, Res. Notes in Math., Vol. 72, Pitman, Boston, Mass., 1982.

11. On derivation ranges and the identity operator, J. Operator Theory 7 (1982), 139-148.

12. $\ldots$, On quasidiagonal weighted shifts and approximation of operators, Indiana Univ. Math. J. 33 (1984), 549-571.

13. Linear and complex analysis problem book (199 Research Problems), Lecture Notes in Math., vol. 1043, Springer-Verlag, 1984.

14. S. Hildebrandt, Uber den numerischen Wertebereich eines operators, Math. Ann. 163 (1966), $230-247$.

15. G. Lumer, Semi-inner-product spaces, Trans. Amer. Math. Soc. 100 (1961), 29-43.

16. J. von Neumann, Eine spektraltheorie fur allgemeine operatoren eines unitaren Raumes, Math. Nachr. 4 (1951), 258-281.

17. C. Pearcy and N. Salinas, Extensions of $C^{*}$-algebras and the reducing essential matricial spectra of an operator, K-theory and operator algebras, (Athens, Georgia, 1975), Lecture Notes in Math., vol. 575, Springer-Verlag, 1977, pp. 96-112. 
18. N. Salinas, Reducing essential eigenvalues, Duke Math. J. 40 (1973), 561-580.

19. R. Schatten, Norm ideals of completely continuous operators, Ergeb. Math. Grenzgeb., Vol. 27, Springer-Verlag, 1960.

20. R. A. Smucker, Quasidiagonal and quasitriangular operators, Dissertation, Indiana University, 1973.

21. J. G. Stampfli, Derivations on $B(H)$ : The range, Illinois J. Math. 17 (1973), 518-524.

22. J. G. Stampfli and J. P. Williams, Growth conditions and the numerical range in a Banach algebra, Tôhoku Math. J. 20 (1968), 417-424.

23. H. Wielandt, Ueber die Unbeschranktheit der Operatoren des Quantenmechanik, Math. Ann. 121 (1949), 21.

24. J. P. Williams, Similarity and the numerical range, J. Math. Anal. Appl. 26 (1969), 307-314.

25. Finite operators, Proc. Amer. Math. Soc. 26 (1970), 129-136.

26. A. Wintner, The unboundedness of quantum-mechanical matrices, Phys. Rev. 71 (1947), 738-739.

27. D. Voiculescu, A non-commutative Weyl-von Neumann theorem, Rev. Roumaine Math. Pures Appl. 21 (1976), 97-113.

Department of Mathematics and Computer Science, State University of New York, College at New Paltz, New Paltz, New York 12561

Department of Mathematics, Arizona State University, Tempe, Arizona 85281 